\title{
Genotypic diversity and variation in mode of reproduction among populations in the Daphnia pulex group
}

David J. Innes, Steven S. Schwartz* and Paul D. N. Hebert
Department of Biology, University of Windsor, Windsor, Ontario, Canada, N9B 3P4.

A survey of nine ponds in Illinois and Iowa confirmed the occurrence of both cyclically and obligately parthenogenetic $D$. pulex and cyclically parthenogenetic $D$. obtusa. The three taxa co-occurred in two of the ponds and one predominantly cyclical parthenogenetic population of $D$. pulex also contained an obligately parthenogenetic clone. Three populations consisted solely of obligately parthenogenetic $D$. pulex, while three consisted solely of cyclically parthenogenetic $D$. obtusa. Populations of cyclically parthenogenetic individuals, which have a sexual phase, were usually in Hardy-Weinberg equilibrium at individual loci and had a high genotypic diversity. Populations of obligately parthenogenetic individuals deviated from Hardy-Weinberg equilibrium and of ten consisted of only one or two clones. Mode of reproduction was confirmed by breeding studies on individuals isolated from the populations. Daphnia pulex and $D$. obtusa were genetically distinct based on variation at six polymorphic loci. Individuals of $D$. pulex reproducing by the two modes of reproduction were also genetically differentiated. Obligately parthenogenetic individuals were Ldh 13 and Pep 12 heterozygotes while cyclically parthenogenetic individuals were 11 homozygotes at both loci. Seven obligately parthenogenetic clones were found with only one clone occurring in more than one population. The high genotypic diversity and restricted distribution of obligately parthenogenetic clones is consistent with their independent origin from cyclically parthenogenetic individuals.

\section{INTRODUCTION}

Parthenogenetic individuals of a species are often found to have a different geographical distribution compared to their bisexual relatives (Levin, 1975; Suomalainen et al., 1976; Glesener and Tilman, 1978; Lynch, 1984a). Asexual forms tend to occur at higher altitudes and latitudes, in xeric environments and disturbed habitats, while sexually reproducing relatives occur in the opposite kinds of habitats (Glesener and Tilman, 1978). Examples of overlapping distributions and the co-occurrence of sexually and asexually reproducing forms of some species show that these patterns are not without exceptions (Lynch, 1984a). Nevertheless, documenting the distribution of parthenogenetic individuals and sexually reproducing relatives is a necessary step towards understanding the origin of parthenogenetic races. For example, the hybrid origin of some parthenogenetic forms from geographically co-occurring or contiguous

\footnotetext{
* Present addres: Department of Biology, University of Houston, Houston, Texas, U.S.A, 77004.
}

bisexual species has been supported by evidence from morphological, cytological, allozyme and mitochondrial DNA analyses (White et al., 1972; Brown and Wright, 1979; Wright et al., 1983). Furthermore, observations on the distribution of parthenogenetic and sexual forms can be used to determine any habitat differences which may favour one mode of reproduction or the other. Although a review of geographic parthenogenesis in terrestrial animals has suggested ecological explanations for the distribution of parthenogenetic and sexual forms (Glesener and Tilman, 1978), no similar comparison has been made for aquatic animal species.

Species of the common planktonic crustacean Daphnia show variation in mode of reproduction (Hebert, 1981). Species of Daphnia typically reproduce by cyclical parthenogenesis. This mode of reproduction involves a parthenogenetic phase followed by a sexual phase in which haploid resting eggs, which require fertilisation to develop, are released into a protective structure known as the ephippium. However recent studies have shown that populations of $D$. pulex in the Canadian arctic 
and Ontario all appear to reproduce by obligate parthenogenesis (Hebert and Crease, 1980; 1983; Hebert and Loaring, 1985). No sexual phase occurs in this mode of reproduction since diploid resting eggs are produced parthenogenetically. The latitudinal distribution of sexual and asexual forms of some species would suggest that cyclically parthenogenetic populations of $D$. pulex may occur further south.

Recently, Lynch (1983) has reported cyclically parthenogenetic $D$. pulex co-occurring with obligately parthenogenetic clones in two Illinois ponds. However, a morphological analysis of invidivuals from one of these ponds has indicated that some of the individuals identified as $D$. pulex in this study, are D. obtusa (Schwartz et al., 1985). Furthermore, individuals of a different genus were accidentally included as a clonal group of $D$. pulex (Lynch, 1984b). Thus, the taxonomic complexity of the $D$. pulex group (Dodson, 1981) requires that the Daphnia taxa from the mid-west U.S. be compared to populations of $D$. pulex from Ontario before the distribution of cyclically parthenogenetic and obligately parthenogenetic forms of this species can be determined. A broader survey of sites was undertaken both to determine the mode of reproduction of the Daphnia taxa in the mid-west U.S. and compare these individuals with clones of $D$. pulex fron Ontario.

\section{MATERIALS AND METHODS}

\section{(a) Collection and electrophoresis}

Nine pounds containing members of the Daphnia pulex group were sampled in Iowa and Illinois during May 1983 (fig. 1). For most samples, 64 clones were derived from individual females and grown separately as previously described (Schwartz et al., 1985). Each clone was assayed electrophoretically for six enzymes; PGI (phosphoglucose isomerase), LDH (lactate dehydrogenase), GOT (glutamate oxaloacetate transaminase), PEP (peptidase), PGM (phosphoglucomutatse) and AMY (amylase). These enzymes were chosen because they were reliably resolved and polymorphic.

PGI, LDH and GOT were stained on a 7 per cent polyacrylamide gel (Young, 1979), PEP was stained on a LiOH starch gel (Selander et al., 1969) and PGM and AMY were stained on cellulose acetate gels (Helena Laboratories, Beaumont, Texas) using a tris-glycine buffer $(3 \mathrm{~g} / 1$ Trizma Base, $14.4 \mathrm{~g} / 1$ glycine). Later studies showed that the PGI, LDH and GOT variants could also be resolved on the cellulose acetate system. Enzyme stains followed standard procedures (Harris and Hopkinson, 1976).

Breeding studies with cyclically parthenogenetic $D$. pulex and $D$. obtusa show that the

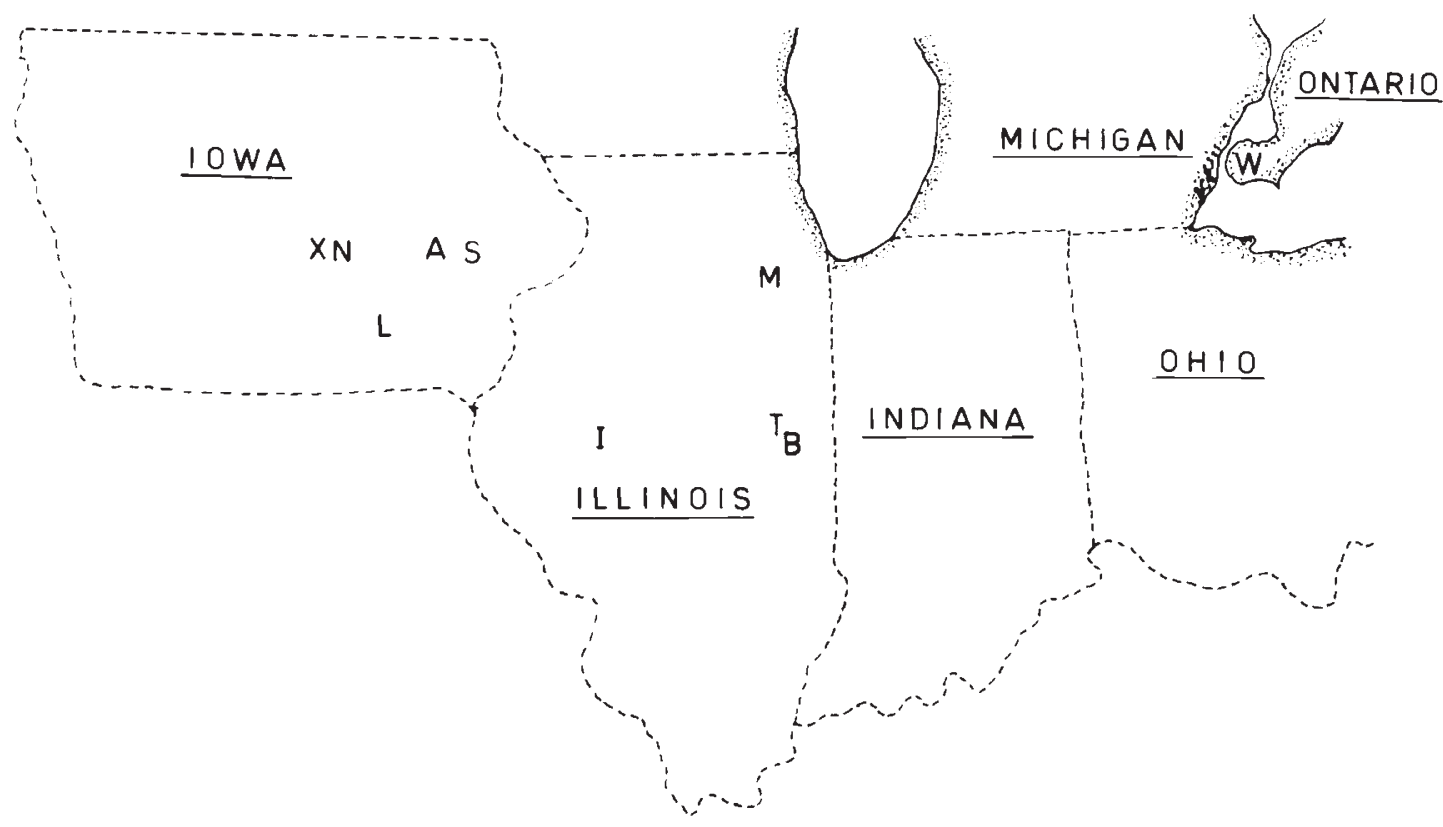

Figure 1 Map of the localities sampled. Localities sampled in Iowa: Baxter (Bax, X), Newton (New, N), Amana (Am, A), Solon (Sol, S), Libertyville (Lib, L). Localities sampled in Illinois: Minooka (Min, M), Idaville (Ida, I), Trelease (Tre, T), Busey (Bu, B). The Windsor (W) Ontario area is shown for reference. 
observed electrophoretic variation is consistent with alleles (electromorphs) segregating at individual loci (Innes, unpubl.). Alleles were numbered in order of increasing mobility and are consistent (except for Amy alleles) with the designations of Hebert and Crease (1983). The Amy locus scored is Amy-1 of Hebert and Crease (1983). Alleles 1, 3, 4 of Hebert and Crease (1983) are designated $1,4,6$, respectively, in this study. Gels stained for PEP showed three zones of activity but only the fastest zone could be reliably scored. This is not the same locus previously scored by Lynch $(1983,1984 b)$.

\section{(b) Population genotypic diversity}

Several approaches were used to test whether individuals in a sampled population were reproducing by cyclical or obligate parthenogenesis. Individual loci which were sufficiently polymorphic (expected value of rarest genotype $>1$ ) were tested for fit to Hardy-Weinberg expectations. Cyclically parthenogenetic populations with frequent sexual reproduction are expected to show a close fit to H.W. expectation (Hebert, 1974b), while obligately parthenogenetic population may show large deviations (Hebert and Crease, 1983). Another test for frequent sexual reproduction in cyclically parthenogenetic populations is to assess genotypic diversity in populations using information on the frequency of multilocus genotypes. Frequent sexual reproduction generates large numbers of multilocus genotypes tinrough recombination, while in populations reproducing by obligate parthenogenesis genic variation is ordinarily tied up in a small number of multilocus genotypes (Hebert and Crease, 1983). Therefore, multilocus genotypic diversity is expected to be decreased by any asexual reproduction. Multilocus genotypic diversity was assessed using two measures; observed genotypic diversity $\left(G_{0}\right)$ and observed number of multilocus genotypes $\left(U_{0}\right)$. Observed genotypic diversity was calculated as:

$$
G_{0}=1 / \sum_{i=1}^{\mathrm{k}} g_{i}^{2}
$$

where $g_{i}$ is the frequency of the $i^{\text {th }}$ multilocus genotype for $k$ multilocus genotypes observed in the sample (Stoddard, 1983). The value of $G_{0}$ varies from 1 , where only a single multilocus genotype is present, to a maximum of $k$ where the $k$ multilocus genotypes are represented in equal frequency.

The values of $G_{0}$ and $U_{0}$ were compared with values expected for a population of sexually repro- ducing individuals. Expected values of genotypic diversity $\left(G_{e}\right)$ and expected number of unique multilocus genotypes $\left(U_{e}\right)$ were generated using computer simulations (Hoffmann, 1986). The simulations used the gene frequencies of the sample and a random number generator to produce random samples of individuals from a simulated sexually reproducing population in HardyWeinberg equilibrium and with free recombination among loci. The same number of individuals were sampled in the simulations as were collected in each of the original population samples. The number of unique multilocus genotypes and the genotypic diversity were calculated for each simulation sample using the formula given above. For each population sample of size $N, 100$ simulation samples of size $N$ were generated and used to calculate a mean and variance for $G_{e}$ and $U_{e}$. The observed values $\left(G_{0}\right.$ and $\left.U_{0}\right)$ were compared to the expected values using a $t$-test for comparing a single observation with a sample mean (Sokal and Rohlf, 1981).

\section{(c) Mode of reproduction}

Determining the mode of reproduction of individuals took advantage of the fact that females of cyclically parthenogenetic clones of Daphnia do not release resting eggs into their ephippium in the absence of males since these eggs require fertilisation to develop. Females of several clones established from single females were kept in isolation of males and the number of empty and filled ephippia counted.

A final test for mode of reproduction of clones from the sampled populations was to determine the genotypes of ephippial hatchlings at loci that were heterozygous in their parent. Clones reproducing by obligate parthenogenesis produce all heterozygous offspring while ephippial hatchlings from cyclically parthenogenetic individuals show segregation at the loci. Ephippial eggs from cyclically parthenogenetic clones, established from single females, are produced by self-fertilisation since parthenogenetically produced males fertilise the eggs (see Bell, 1982, page 29).

\section{(d) Genetic relatedness}

Multilocus genotypes (based on the six loci) of Daphnia obtusa and D. pulex were compared using a simple matching coefficient (Sneath and Sokal, 1973). For a pair of multilocus genotypes, compared at a locus, a coefficient of similarity of 0 , 0.5 or 1 was computed if the two multilocus 
genotypes had 0,1 or 2 alleles in common, respectively. This coefficient of similarity was then averaged over the six loci and converted to Euclidean distance by taking the square root of the complement of the coefficient (Sneath and Sokal, 1973). Principal coordinate analysis (Gower, 1966) of the distance matrix was used to summarize the genetic differences among the multilocus genotypes of the two species. Principal coordinate analysis was also used to compare genetic differences between cyclically parthenogenetic and obligately parthenogenetic D. pulex.

\section{RESULTS}

\section{(a) Single locus variation}

Daphnia obtusa was present in samples from five of the nine sites, while $D$. pulex was collected from six sites. Tables 1 and 2 list the genotype frequencies observed from samples of each species. Goodness-of-fit tests indicated that Daphnia obtusa from three populations ( $\mathrm{Bax}, \mathrm{New}, \mathrm{Bu}$ ) had genotype frequencies consistent with HardyWeinberg expectations (table 3 ). The sample from Lib was monomorphic at all six loci and too few individuals of this species were sampled from the Tre population to be tested. In contrast to these results, five of the $D$. pulex populations showed significant deviations from Hardy-Weinberg expectations at 1-4 loci (table 3). Deviations in samples from the Min, Sol and Ida populations were due to fixed heterozygotes at several loci or in the case of the Tre sample to an excess of heterozygotes. The $\mathrm{Bu}$ population showed no significant deviation at the Amy locus, but a significant deviation at the Pgm locus while the Am population was not sufficiently polymorphic to be tested.

\section{(b) Multilocus genotypic diversity}

The observed number of multilocus genotypes and multilocus genotypic diversity were calculated for each sample (table 4). These were compared to the values expected for populations of sexually reproducing individuals (table 4) derived from a computer simulation as explained in the methods section. No significant differences were detected between observed and expected values for the three polymorphic populations of $D$. obtusa (Bax, New, $\mathrm{Bu})$. Two samples of $D$. pulex $(\mathrm{Am}, \mathrm{Bu})$ also showed no significant differences between observed and expected values. However, four samples of $D$. pulex (Tre, Min, Sol, Ida) had a significantly reduced genotypic diversity and a smaller number of multilocus genotypes compared to the expected values (table 4).

\section{(c) Mode of reproduction}

Fourteen clones of $D$. obtusa, established from single females from three samples (Bax, New, Bu), were tested for mode of reproduction using observations on the ephippial hatchlings. The clones used were heterozygous at one to three loci, and produced ephippial eggs by intraclonal matings. Segregation of genotypes in the ephippial hatchlings was found for all 14 clones (table 5) with only six of the 28 tests at individual loci showing a significant deviation from the expected $1: 2: 1$ ratio of the genotypes. Of the 16 clones of D. pulex established from single females (table 6), four showed segregation ( 3 from $\mathrm{Am}, 1$ from $\mathrm{Bu}$ ) while 12 did not ( 3 from Tre, 1 from Bu, 3 from Ida, 3 from Min, 2 from Am). Lack of segregation of the marker loci in ephippial hatchlings is evidence that these clones reproduce by obligate parthenogenesis. Individuals from the Sol population failed to produce ephippia in culture and therefore could not be tested.

Table 1 Genotype frequencies for samples of Daphnia obtusa. All samples were fixed for the 1 allele at $L d h$ and $P e p$

\begin{tabular}{|c|c|c|c|c|c|c|c|c|c|c|c|c|c|c|}
\hline \multirow[b]{3}{*}{ Locality } & \multirow[b]{3}{*}{$\mathrm{N}$} & \multicolumn{13}{|c|}{ Locus } \\
\hline & & \multicolumn{3}{|c|}{$P g i$} & \multicolumn{3}{|c|}{ Got } & \multicolumn{3}{|c|}{ Amy } & \multicolumn{4}{|c|}{ Pgm } \\
\hline & & 11 & 14 & 44 & 11 & 12 & 22 & 22 & 25 & 55 & 23 & 33 & 34 & 44 \\
\hline $\mathrm{Bax}$ & 64 & 64 & 0 & 0 & 40 & 22 & 2 & 15 & 29 & 20 & 0 & 64 & 0 & 0 \\
\hline New & 64 & 64 & 0 & 0 & 16 & 27 & 21 & 35 & 26 & 3 & 8 & 53 & 3 & 0 \\
\hline Lib & 64 & 64 & 0 & 0 & 0 & 0 & 64 & 0 & 0 & 64 & 0 & 64 & 0 & 0 \\
\hline $\mathrm{Bu}$ & 22 & 19 & 1 & 2 & 7 & 10 & 5 & 12 & 9 & 1 & 0 & 8 & 11 & 3 \\
\hline Tre & 8 & 8 & 0 & 0 & 7 & 1 & 0 & 8 & 0 & 0 & 0 & 8 & 0 & 0 \\
\hline
\end{tabular}


Table 2 Genotype frequencies for samples of Daphnia pulex

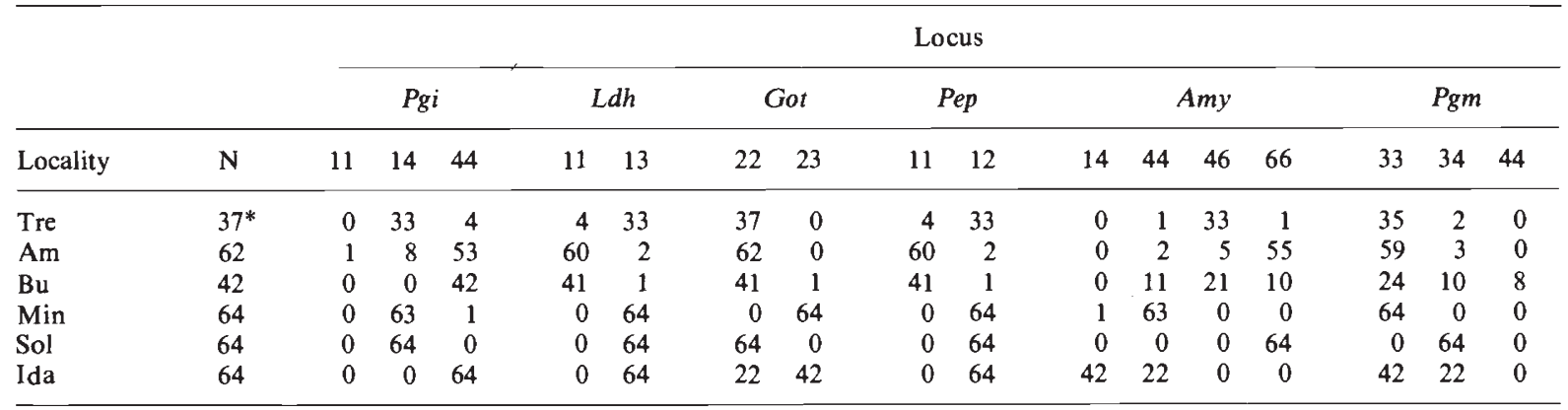

* Two individuals not scored for Amy

Additional evidence on the mode of reproduction of clones derived from single females was obtained by examining ephippia produced in the absence of males (table 7). Seven clones (Am 22, Bu 60, Ida 6, 7, Tre 16, 17, Min 3) released resting eggs into their ephippia providing evidence for reproduction by obligate parthenogenesis. Three clones (Am 64, Bu 19, Tre 43) released empty ephippia, suggesting that these clones reproduce by cyclical parthenogenesis. In addition, $\mathrm{Bu} 19$ and Tre 43 females have been used in crossing experiments and are known to reproduce by cyclical parthenogenesis (Innes, unpubl.).

\section{(d) Genetic relatedness}

Fig. 2 is a plot of the first two principal coordinate axes (explaining 51 per cent of the total variation) comparing multilocus genotypes of $D$. pulex and D. obtusa. The two species are almost totally separated along the first axis. No Amy alleles were shared among the two species with variation at Pgi and Got also contributing to this separation (tables 1 and 2). These results show that in addition to morphological differences (Schwartz et al., 1985), $D$. pulex and D. obtusa are genetically distinct with no eviderce for hybridisation between them.

Fig. 3 compares multilocus genotypes of Daphnia pulex using principal coordinate analysis. The plot of the first two axes (explaining 56 per cent of the total variation) shows that multilocus genotypes from the mid-west U.S. reproducing by cyclical parthenogenesis are genetically distinct from multilocus genotypes reproducing by obligate parthenogenesis. Two loci were primarily responsible for this separation. Obligately parthenogenetic clones were $L d h 13$ and Pep 12 heterozygotes while cyclically parthenogenetic individuals were $L d h 11$ and Pep 11 homozygotes (tables 6, 7 and 8). When five obligately parthenogenetic clones from southern Ontario (Hebert and Crease, 1983) were plotted (fig. 3),

Table 3 Tests for fit to Hardy-Weinberg expectation for samples of Daphnia pulex and $D$. obtusa using chi-square goodness of fit. A dash indicated the locus was not sufficiently polymorphic to test. Chi-square values $>3.8$ are significant at $p<0.05$

\begin{tabular}{|c|c|c|c|c|c|c|}
\hline & \multicolumn{6}{|c|}{ Locus } \\
\hline & $P g i$ & $L d h$ & Got & $P e p$ & Amy & Pgm \\
\hline \multicolumn{7}{|c|}{ Daphnia pulex } \\
\hline Tre & $24 \cdot 0$ & $24 \cdot 0$ & - & $24 \cdot 0$ & $27 \cdot 5$ & - \\
\hline $\mathrm{Am}$ & - & - & - & - & - & - \\
\hline $\mathrm{Bu}$ & - & - & - & - & $0 \cdot 0$ & $8 \cdot 2$ \\
\hline Min & $60 \cdot 1$ & $64 \cdot 0$ & $64 \cdot 0$ & $64 \cdot 0$ & $60 \cdot 1$ & - \\
\hline Sol & $64 \cdot 0$ & $64 \cdot 0$ & - & $64 \cdot 0$ & - & $64 \cdot 0$ \\
\hline Ida & - & $64 \cdot 0$ & $15 \cdot 3$ & $64 \cdot 0$ & $15 \cdot 3$ & - \\
\hline \multicolumn{7}{|c|}{ Daphnia obtusa } \\
\hline Bax & - & - & 0.2 & - & 0.5 & - \\
\hline New & - & - & 1.5 & - & 0.4 & - \\
\hline Lib & - & - & - & - & - & - \\
\hline $\mathrm{Bu}$ & - & - & 0.2 & - & - & $0 \cdot 1$ \\
\hline Tre & - & - & - & - & - & - \\
\hline
\end{tabular}


Table 4 Genetic diversity fer samples of Daphnia obtusa and Daphnia pulex. Observed genotypic diversity $\left(G_{0}\right)$ and expected $\left(G_{e}\right)$ based on computer simulation. Observed number $\left(U_{0}\right)$ of multilocus genotypes and expected number $\left(U_{e}\right)$ based on computer simulation. Observed and expected values were tested for significant differences using a $t$-test (see materials and methods)

\begin{tabular}{lcrrrrrr}
\hline & $N$ & \multicolumn{1}{c}{$G_{0}$} & \multicolumn{1}{c}{$G_{e}$} & $G_{0} / G_{e}$ & $U_{0}$ & \multicolumn{1}{c}{$U_{e}$} & \multicolumn{1}{c}{$U_{0} / U_{e}$} \\
\hline Daphnia obtusa & & & & & & & \\
Bax & 64 & 5.46 & 4.92 & 1.11 & 8 & 7.61 & 1.05 \\
New & 64 & 7.94 & 7.35 & 1.08 & 15 & 15.56 & 0.96 \\
Bu & 22 & 13.44 & 11.73 & 1.15 & 16 & 14.95 & 1.07 \\
Daphnia pulex & & & & & & & \\
Tre & 37 & 1.25 & 22.71 & $0.06^{*}$ & 4 & 27.73 & $0.14^{*}$ \\
Am & 62 & 1.52 & 2.17 & 0.70 & 8 & 8.00 & 1.00 \\
Bu & 42 & 6.58 & 6.41 & 1.03 & 10 & 10.47 & 0.96 \\
Min & 64 & 1.03 & 43.41 & $0.02^{*}$ & 2 & 51.85 & $0.04^{*}$ \\
Sol & 64 & 1.00 & 28.76 & $0.03^{*}$ & 1 & 39.32 & $0.03^{*}$ \\
Ida & 64 & 1.82 & 31.28 & $0.06^{*}$ & 2 & 42.32 & $0.05^{*}$ \\
\hline
\end{tabular}

$* p<0.001$

three (W1, W4, W12) were found to be genetically similar to the obligately parthenogenetic clones sampled from the mid-west U.S. (table 8) while two (W8, W13) were genetically similar to individuals sampled from cyclically parthenogenetic populations in the mid-west U.S.

\section{DISCUSSION}

Cyclically parthenogenetic and obligately parthenogenetic Daphnia pulex and cyclically parthenogenetic $D$. obtusa occur in the mid-west U.S. This contrasts with previous surveys in the

Table 5 Mode of reproduction in clones of Daphnia obtusa. Cyclically parthenogenetic clones show segregation of genotypes in ephippial hatchlings derived from intraclonal matings of a heterozygous parent. $N$ is the number of ephippial hatchlings examined for each clone

\begin{tabular}{llrc}
\hline & Heterozygous & & \\
Clone & locus & $N$ & Segregation \\
\hline Bu 3 & Pgm, Amy, Got & 33 & Yes \\
Bu 24 & Pgm, Amy & 59 & Yes \\
Bu 2 & Pgm & 35 & Yes \\
Bu 28 & Pgm, Got & 38 & Yes \\
Bu 29 & Got, Amy & 69 & Yes \\
Bu 33 & Pgm, Amy, Got & 23 & Yes \\
Bu 37 & Pgm, Got & 40 & Yes \\
Bu 54 & Pgm, Amy & 21 & Yes \\
Bax 20 & Got, Amy & 48 & Yes \\
Bax 28 & Amy & 156 & Yes \\
Bax 41 & Got, Amy & 89 & Yes \\
Bax 56 & Got,Amy & 119 & Yes \\
New 45 & Pgm, Amy & 68 & Yes \\
New 63 & Got, Amy & 31 & Yes \\
\hline
\end{tabular}

Canadian arctic and Ontario where only obligately parthenogenetic clones of $D$. pulex were found (Hebert and Crease, 1983; Hebert and Loaring, 1985). Recently Lynch (1983) has reported two cyclically parthenogenetic clonal groups of $D$. pulex from Illinois. However, the composite genotype data in table 1 of Lynch (1983) suggest that some of the individuals classified as group B are D. obtusa. A direct comparison of the genotypes identified in this previous study with the present samples was not possible since only three loci ( Pgi, $P g m, G o t)$ were common to both studies. However, individuals identified as members of group B (Lynch per. com.) were found to be morphologically and genetically similar to $D$. obtusa. Obligately parthenogenetic and cyclically parthenogenetic $D$. pulex as well as cyclically parthenogenetic $D$. obtusa appear to be coexisting in Busey Pond. The complex morphological variation observed for individuals in the Daphnia pulex group (Dodson, 1981) has been interpreted to be "... a consequence of rampant speciation." (Lynch, 1983, p. 370). Alternatively, morphological complexity in this group may in part be due to the failure to distinguish morphological variation associated with distinct species (such as $D$. obtusa) and variation among different clones of D. pulex (Schwartz et al., 1985).

$\mathrm{Bu}$ and Tre were the only two populations in which cyclically parthenogenetic and obligately parthenogenetic $D$. pulex were found together with $D$. obtusa. Only D. pulex was sampled from Am which appeared to be a predominantly cyclically parthenogenetic population, although one obligately parthenogenetic clone was found. Samples from the remaining six populations consisted of 
Table 6 Mode of reproduction in clones of Daphnia pulex. Cyclically parthenogenetic clones show segregation of genotypes in ephippial hatchlings derived from a heterozygous parent. Lack of segregation is evidence for obligately parthenogenetic reproduction. $N$ is the number of ephippial hatchlings examined for each clone. $P$ is the probability of obtaining by chance no segregation (all heterozygous hatchlings) in a sexually reproducing clone

\begin{tabular}{|c|c|c|c|c|c|c|}
\hline \multirow[b]{2}{*}{ Clone } & \multirow{2}{*}{$\begin{array}{l}\text { Heterozygous } \\
\text { locus }\end{array}$} & \multicolumn{2}{|c|}{ Genotype } & \multirow[b]{2}{*}{$N$} & \multirow[b]{2}{*}{ Segregation } & \multirow[b]{2}{*}{$P$} \\
\hline & & $L d h$ & Pep & & & \\
\hline Tre 16 & Ldh, Pgi, Amy & 13 & 12 & 24 & No & $<0.001$ \\
\hline Tre 21 & $A m y, L d h, P g i$ & 13 & 12 & 7 & No & $<0.001$ \\
\hline Am 22 & Ldh, Pgm & 13 & 12 & 24 & No & $<0.001$ \\
\hline Am 46 & $L d h, P g m$ & 13 & 12 & 24 & No & $<0.001$ \\
\hline Am 54 & $P g i$ & 11 & 11 & 3 & Yes & - \\
\hline Ida 6 & Ldh, Pgm & 13 & 12 & 24 & No & $<0.001$ \\
\hline Ida 33 & $L d h, G o t$ & 13 & 12 & 21 & No & $<0.001$ \\
\hline Ida 55 & $L d h$ & 13 & 12 & 7 & No & $<0.01$ \\
\hline Min 3 & $L d h, G o t$ & 13 & 12 & 24 & No & $<0.001$ \\
\hline $\operatorname{Min} 7$ & $L d h, P g i$, Got & 13 & 12 & 18 & No & $<0.001$ \\
\hline
\end{tabular}

only obligately parthenogenetic D. pulex (Sol, Min, Ida) or cyclically parthenogenetic D. obtusa (New, Bax, Lib). Although most populations appeared to be in temporary habitats lasting no more than several weeks, successive samples would be required to determine if the composition of these populations changed over the course of a season.

Results from this study show that the mode of reproduction of individuals making up populations of Daphnia can often be determined from information on the distribution of genetic variation within populations. Cyclically parthenogenetic populations are usually in Hardy-Weinberg equilibrium at individual loci and have a high genotypic

Table 7 Number of eggs found in ephippia of Daphnia pulex females isolated from males

\begin{tabular}{|c|c|c|c|c|c|c|}
\hline \multirow[b]{2}{*}{ Clone } & \multicolumn{2}{|c|}{ Genotype } & \multirow[b]{2}{*}{$N$} & \multicolumn{3}{|c|}{$\begin{array}{c}\text { Number of eggs in } \\
\text { Ephippia }\end{array}$} \\
\hline & $L d h$ & Pep & & 0 & 1 & 2 \\
\hline Am 22 & 13 & 12 & 17 & 1 & 3 & 13 \\
\hline Am 64 & 11 & 11 & 63 & 63 & 0 & 0 \\
\hline Bu 19 & 11 & 11 & 127 & 127 & 0 & 0 \\
\hline $\mathrm{Bu} 60$ & 13 & 12 & 45 & 4 & 8 & 33 \\
\hline Ida 6 & 13 & 12 & 41 & 3 & 8 & 30 \\
\hline Ida 7 & 13 & 12 & 32 & 4 & 6 & 22 \\
\hline Tre 16 & 13 & 12 & 17 & 1 & 2 & $14^{*}$ \\
\hline Tre 17 & 13 & 12 & 17 & 0 & 0 & 17 \\
\hline Tre 43 & 11 & 11 & 128 & 128 & 0 & 0 \\
\hline $\operatorname{Min} 3$ & 13 & 12 & 49 & 3 & 10 & 36 \\
\hline
\end{tabular}

* includes two ephippia with three eggs. diversity. This is true of populations in temporary habitats where periods of parthenogenetic reproduction are frequently interrupted by sexual reproduction (Hebert, 1974b). Populations composed of obligate parthenogens are characterised by individual loci deviating from Hardy-Weinberg equilibrium and a low genotypic diversity (Hebert and Crease, 1983). However, these characteristics are also found for populations of cyclical parthenogens in more permanent habitats where periods of sexual reproduction may be much less frequent (Herbert, 1974a; but see Mort and Wolf, 1985 for an exception). Furthermore, populations composed of both cyclically parthenogenetic and

Table 8 Six-locus genotypes of obligately parthenogenetic Daphnia pulex from the mid-west U.S. and five obligately parthenogenetic clones from Windsor, Ontario (W)

\begin{tabular}{lllllll}
\hline \multicolumn{7}{c}{ Locus } \\
\cline { 1 - 4 }$P g i$ & Ldh & Got & Pep & Amy & Pgm & Population \\
\cline { 1 - 4 } 14 & 13 & 22 & 12 & 46 & 33 & Tre \\
14 & 13 & 22 & 12 & 44 & 34 & Am \\
14 & 13 & 23 & 12 & 44 & 33 & Min \\
44 & 13 & 23 & 12 & 14 & 33 & Min, Ida \\
14 & 13 & 22 & 12 & 66 & 34 & Sol \\
44 & 13 & 22 & 12 & 44 & 34 & Ida \\
44 & 13 & 23 & 12 & 44 & 33 & Bu \\
44 & 13 & 22 & 12 & 46 & 22 & W1 \\
11 & 13 & 22 & 12 & 46 & 33 & W4 \\
44 & 13 & 22 & 12 & 14 & 33 & W12 \\
44 & 11 & 22 & 11 & 66 & 34 & W8 \\
44 & 11 & 22 & 11 & 66 & 33 & W13 \\
\hline
\end{tabular}




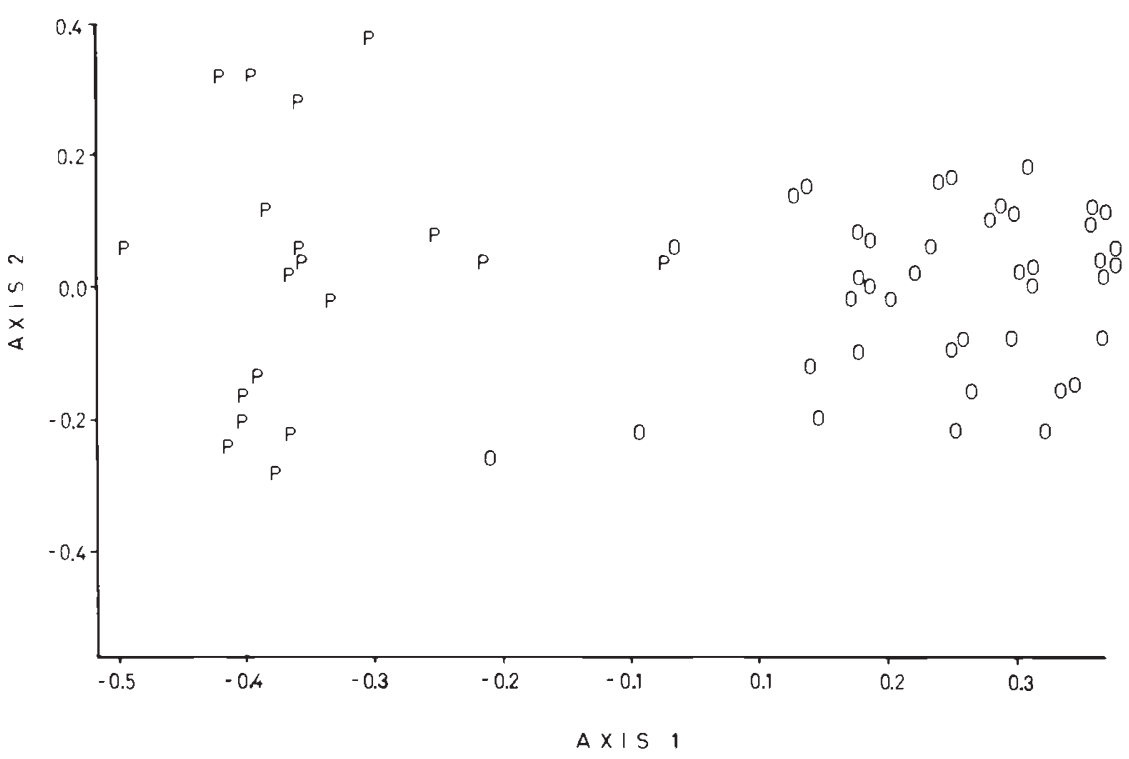

Figure 2 Principal coordinate plot (first 2 principal axes of individuals of Daphnia pulex (P) and D. obtusa (O) based on six-locus genotypes.

obligately parthenogenetic individuals may often appear to conform to one of the two modes of reproduction. For example, the sample of $D$. pulex from the Tre population showed large deviations from Hardy-Weinberg expectation at four loci (table 3 ) suggesting that the population was composed of only obligate parthenogens. However, observations on the mode of reproduction of individuals revealed at least one individual reproducing by cyclical parthenogenesis. Daphnia pulex sampled from the $\mathrm{Bu}$ and $\mathrm{Am}$ populations conformed to the genetic characteristics expected for populations reproducing by cyclical parthenogenesis with frequent periods of sexual reproduction. However, one obligately parthenogenetic clone in the Am population

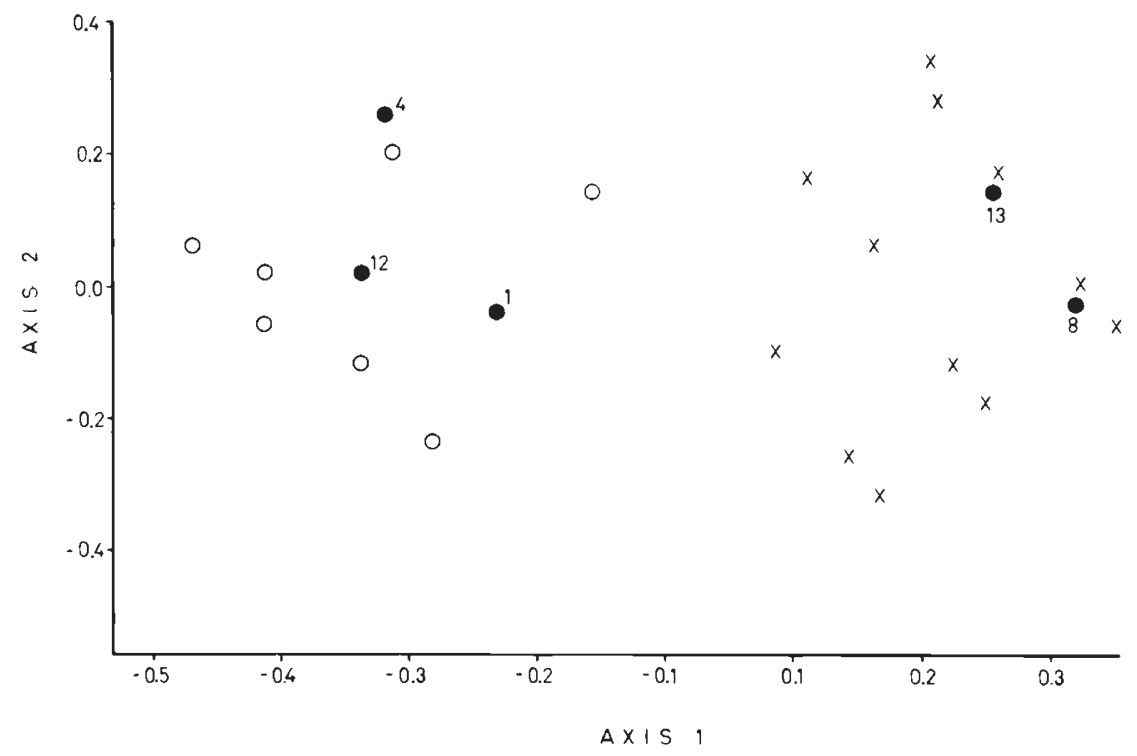

Figure 3 Principal coordinate plot of individuals of Daphnia pulex based on six-locus genotypes. Open circles represent obligately parthenogenetic individuals while individuals from cyclically parthenogenetic populations are represented by $\mathrm{X}$. Closed circles are obligately parthenogenetic clones W1, W4, W8, W12 and W13 from southern Ontario (Hebert and Crease, 1983). 
sample and one in the $\mathrm{Bu}$ population sample were identified by determining the mode of reproduction of a sample of individuals from each population. Therefore, the reproductive characteristics of populations of Daphnia, determined using information on allozyme variation, should be supplemented by direct observation on the mode of reproduction of individuals. It should also be pointed out that the presence of males in a population can not be used to identify cyclically parthenogenetic populations. Some obligately parthenogenetic clones are capable of producing males (Hebert and Crease, 1983) even though they are not necessary for the production of resting eggs by these clones.

The obligately parthenogenetic and cyclically parthenogenetic $D$. pulex sampled in the present study were found to be genetically distinct with $L d h$ and $P e p$ potential markers for discriminating individuals reproducing by the two modes. Cyclically parthenogenetic individuals were 11 homozygotes at both loci while obligately parthenogenetic individuals were $L d h 13$ and $P e p 12$ heterozygotes. The two $L d h$ genotypes were also associated with genetically distinct groups of clones in samples from Ontario (Hebert and Crease, 1983). However, all of these clones appeared to be reproducing by obligate parthenogenesis. Comparing the clones sampled in the mid-west U.S. and Ontario (fig. 3) shows that $D$. pulex is made up of at least two groups of obligate parthenogenetic clones and a group of cyclically parthenogenetic individuals. One group of obligate parthenogens (with the $L d h$ and Pep 11 genotype) occurring in Ontario, was not present in the samples from the mid-west U.S. However, the group of obligate parthenogens with the Ldh 13 and Pep 12 genotype was common in both areas. In the mid-west U.S. samples, this group was composed of seven clones based on variation at six loci (table 8 ). Only one of these clones was found in more than one population and none of the reference clones from Ontario occurred in any of the mid-west populations. This extremely restricted distribution was also found for clones sampled in Ontario (Hebert and Crease, 1983).

Similar to the sexual relatives of other parthenogentic species (Suomalainen et al., 1976), cyclically parthenogenetic Daphnia pulex appear to occupy a more restricted range than the obligate parthenogens. Obligately parthenogenetic clones occur in arctic Canada, Ontario and the mid-west U.S. while samples to date show cyclically parthenogenetic populations only rarely in Ontario but more commonly in Michigan (unpublished observations), Illinois and Iowa. Some eastern U.S. populations (Berger and Sutherland, 1978) are probably composed of obligate parthenogens (Hebert, and Crease, 1983). Although D. pulex is common throughout North America (Brooks, 1957), the mode of reproduction of populations further west or south is presently unknown.

Obligately parthenogenetic $D$. pulex from the mid-west U.S. and Ontario (Hebert and Crease, 1983) form a genotypically diverse group of clones. This suggests that these clones have originated independently from cyclically parthenogenetic ancestors. It has been proposed that males from obligately parthenogenetic clones may have mated with sexual females from cyclically parthenogenetic populations and produced genetically diverse obligately parthenogenetic progeny (Hebert and Crease, 1983). This may be possible since some obligately parthenogenetic clones are capable of producing males (Hebert and Crease, 1983). Furthermore, crosses between these males and sexual females from cyclically parthenogenetic populations have recently been shown to produce obligately parthenogenetic progeny (Innes and Hebert, in prep.). These matings may continue to generate new obligately parthenogenetic clones in natural populations and would eventually eliminate cyclically parthenogenetic individuals from the population (Hebert, 1983).

The high heterozygosity of obligately parthenogenetic clones of $D$. pulex compared to cyclically parthenogenetic populations was thought to derive from the accumulation of mutations over many generations (Hebert and Crease, 1983). Alternatively, highly heterozygous clones could be generated if males from obligately parthenogenetic clones mated with closely related but genetically differentiated cyclically parthenogenetic females. For example, many obligately parthenogenetic clones are $L d h 13$ and $P e p$ 12 heterozygotes but all cyclically parthenogenetic populations surveyed to date are monomorphic for the 1 allele at these loci. A cyclically parthenogenetic population has recently been found in which the $L d h 3$ and Pep 2 alleles are present (Innes, unpubl.). Highly heterozygous individuals have been produced in crosses between males from obligately parthenogenetic clones and sexual females from this population (Innes, unpubl.). Similar matings may have given rise to some of the highly heterozygous obligately pathenogenetic clones observed in natural populations.

Most parthenogenetic organisms have geographically widespread clones (Lynch, 1984a). The situation is different for $D$. pulex where the wide geographic distribution of obligate 
parthenogenesis is clearly due to many clones with a restricted distribution rather than a few broadly distributed clones. This can be explained by the mode of origin of new clones and the restricted dispersal capabilities of Daphnia. Although a multiple origin will generate many new clones from cyclically parthenogenetic populations, most of this variation appears to be between populations. The small number of clones within each pond may be due to the selection of clones adapted to local conditions. However, no information is available to distinguish this explanation from chance events reducing the clonal diversity within ponds.

The geographical distribution of cyclical and obligate parthenogens of Daphnia pulex may be a consequence of the contagious spread of a gene or genes suppressing meiosis in females rather than environmental differences favouring one mode of reproduction, or the other. This is supported by the observation that cyclically and obligately parthenogenetic forms rarely co-occur but appear to occupy environmentally similar ponds. The persistence of cyclically parthenogenetic populations in the mid-west U.S. may be due to the low dispersal capability of the obligately parthenogenetic clones retarding the spread of obligate parthenogenesis and delaying the eventual extinction of cyclically parthenogenetic populations. There is also evidence that additional factors could be responsible for the persistence of cyclically parthenogenetic individuals. For example, not all obligately parthenogenetic clones produce males and some of these clones appear to coexist with cyclically parthenogenetic individuals (Lynch, 1983). The ability to produce functional males by an obligately parthenogenetic clone may be lost by the time it has dispersed to a pond of cyclically parthenogenetic individuals. Furthermore, the exact genetic mechanism responsible for the suppression of meiosis in females has not been determined. It is possible that the genetic control of mode of reproduction produces some obligate parthenogens when males from obligately parthenogenetic clones mate with cyclically parthenogenetic females but does not result in a contagious spread of the genes suppressing meiosis. Also, some cyclically parthenogenetic clones may have a genotype which blocks the expression of the meiosis suppressing genes in progeny resulting from a cross with males from obligately parthenogenetic clones. Studies are continuing on the genetic basis of obligate parthenogenesis (Innes and Hebert, in prep.) and the distribution of obligate and cyclical parthenogenesis in southern Ontario and
Michigan. This information will be used to determine the origin of obligately parthenogenetic clones and the factors responsible for the persistence of cyclically parthenogenetic populations.

Acknowledgements We are grateful to L. Harshman, R. Hoffmann, M. Lynch and L. Weider for comments on the manuscript. This study was supported by a N.S.E.R.C. Postdoctoral Fellowship to D.J.I. and a N.S.E.R.C. Grant to P.D.N.H.

\section{REFERENCES}

BELL, G. 1982. The Masterpiece of Nature. Univ. Calif. Press, Berkeley.

BERGER, E. AND SUTHERLAND, J. 1978. Allozyme variation in two natural populations of Daphnia pulex. Heredity, 41, $13-23$.

BROOKS, J. L. 1957. The systematics of North American Daphnia. Mem. Conn. Acad. Arts Sci., 13, 1-180.

BROWN, W. M. AND WRIGHT, J. W. 1979. Mitochondrial DNA analyses and the origin and relative age of parthenogenetic lizards (Genus Cnemidophorus). Science, 203, 1247-1249.

DODSON, S. I. 1981. Morphological variation of Daphnia pulex Leydig (Crustacea: Cladocera) and related species from North America. Hydrobiologia, 83, 101-114.

GLESENER, R. R. AND TILMAN, D. 1978. Sexuality and the components of environmental uncertainty: Clues from geographic parthenogenesis in terrestrial animals. Amer. Natur., 112, 659-673.

GOWER, J. C. 1966. Some distance properties of latent roots and vector methods used in multivariate analysis. Biometrika, 53, 325-338.

HARRIS H. AND HOPKINSON, D. A. 1976. Handbook of Enzyme Electrophoresis in Human Genetics. North Holland, Amsterdam.

HEBERT, P. D. N. 1974a. Enzyme variability in natural populations of Daphnia magna. II Genotypic frequencies in permanent populations. Genetics, 77, 323-334.

HEBERT, P. D. N. 1974b. Enzyme variability in natural populations of Daphnia magna. III Genotypic frequencies in intermittent populations. Genetics, 77, 335-341.

HEBERT, P. D. N. 1983. Clonal diversity in cladoceran populations. In King, C. E. and Dawson, P. S. (eds.) Population Biology: Retrospective and Prospect, Columbia University Press, New York. p. 37-59.

HEBERT, P. D. N. AND CREASE, T. J. 1980. Clonal coexistence in Daphnia pulex (Leydig): another planktonic paradox. Science, 207, 1363-1365.

HEBERT, P. D. N. AND CREASE, T. J. 1983. Clonal diversity in populations of Daphnia pulex reproducing by obligate parthenogenesis. Heredity, 51, 353-369.

HEBERT, P. D. N. AND LOARING, J. M. 1985. Systematics of the Daphnia pulex group: Variation in the agamic complex and description of a species new to North America. Biochem. Syst. Ecol., 13, 333-340

HOFFMANN, R. J. 1986. Variation in contributions of asexual reproduction to the genetic structure of populations of the sea anemone Metridium senile. Evolution, 40, 357-365.

LEVIN, D. A. 1975. Pest pressure and recombination systems in plants. Amer. Natur., 109, 437-452.

LYNCH, M. 1983. Ecological genetics of Daphnia pulex. Evolution, 37, 358-374. 
LYNCH, M. 1984a. Destabilizing hybridizatiori, general-purpose genotypes and geographic parthenogenesis. Quart. Rev. Biol., 59, 257-290.

LYNCH, M. 1984b. The genetic structure of a cyclical parthenogen. Evolution, 38, 186-203.

MORT, M. A. AND WOLF, H. G. 1985 . Enzyme variability in large-lake Daphnia populations. Heredity, 55, 27-36.

SCHWARTZ, S. S., INNES, D. J. AND HEBERT, P. D. N. 1985. Morphological separation of Daphnia pulex and Daphnia obtusa in North America. Limnol. Oceanogr., 30, 189- 197.

SElANDER, R. K., YANG, S. Y. AND HUNT, W. G. 1969. Polymorphism in esterases and hemoglobin in wild populations of the house mouse (Mus musculus). Studies in Genetics V. Univ. Texas Publ., 6918, 271-338.

SNEATH, P. H. A. AND SOKAL, R. R. 1973. Numerical Taxonomy. W. H. Freeman and Co., San Francisco.
SOKAL, R. R. AND ROHLF, F. J. 1981. Biometry (2nd edn.). W. H. Freeman and Co., San Franscico.

STODDARD, J. A. 1983. A genotypic diversity measure. $J$. Heredity, 74, 489-490.

SUOMALAINEN, E., SAURA, A. AND LOKKI, J. 1976. Evolution of parthenogenetic insects. Evol. Biol., 9, 209-257.

WHITE, M. J. D., CONTRERAS, N., CHENEY, J. AND WEBB, G. C. 1977. Cytogenetics of the parthenogenetic grasshopper Warramaba (formerly Moraba) virgo and its bisexual relatives. II. Hybridization stuciies. Chromosoma, 61, 127-148.

WRIGHT, J. W., SPOLSKY, C. AND BROWN, W. M. 1983. The origin of the parthenogenetic lizard Cnemidophorus laredoensis inferred from mitochondrial DNA analysis. Herpetologica, 39, 410-416.

YOUNG, J. P. W. 1979. Enzyme polymorphism and cyclical parthenogenesis in Daphnia magna. I. Selection and clonal diversity. Genetics, 92, 953-870. 